\title{
Numerical Calculation of Adiabatic Corrections with the Born-Handy Method*
}

\author{
Krzysztof Strasburger \\ Institute of Physical and Theoretical Chemistry \\ Wroclaw University of Technology \\ Wybrzeże Wyspiańskiego 27, 50-370 Wroctaw, Poland \\ e-mail: strasbur@chkw386.ch.pwr.wroc.pl
}

(Rec. June 12, 2006)

\begin{abstract}
A modified algorithm of the numerical calculation of adiabatic corrections is proposed. It is based on earlier approaches, introduced by Cencek and Kutzelnigg and by the present author. The adiabatic correction is approximated in terms of overlap integrals between electronic wave functions for a given geometry and for a single nucleus shifted by $h$. The leading term of error is proportional to the square of $h$ in the original methods. The new approach, while requires the same wave functions as those methods, shrinks the error, so that it becomes proportional to $h^{4}$ in atomic cases. Test calculations show, that similar behavior is retained also for two-atom molecules and additional stable decimal digit of the adiabatic correction can be obtained.
\end{abstract}

Key words: adiabatic correction, explicitly correlated Gaussian function, Born-Handy method

\section{INTRODUCTION}

The separation of nuclear motions within the adiabatic approximation is a foundation of almost all quantum chemical calculations. Following the textbooks of quantum chemistry, the wave function is assumed in the form of a product of functions describing the nuclei (which coordinates will be denoted by $\mathbf{R}$ ) and light particles (with coordinates denoted by $\mathbf{r}$ ):

$$
\Psi(\mathbf{R}, \mathbf{r})=\Psi_{n}(\mathbf{R}) \Psi_{l}(\mathbf{R}, \mathbf{r}) .
$$

The function $\Psi_{l}$ is usually called as the electronic function, but it is also obvious to treat the positrons on equal footing with electrons, as their masses are the same and they often move in the same time scale. As both electrons and positrons are leptons and wave functions for positronic atoms and molecules will be used in calculations presented in this article, $\Psi_{l}$ will be named as the leptonic function.

The nuclear kinetic energy operator $\left(\hat{T}_{n}\right)$ is separated out of the hamiltonian

$$
\hat{H}(\mathbf{R}, \mathbf{r})=\hat{T}_{n}(\mathbf{R})+\hat{H}_{l}(\mathbf{R}, \mathbf{r})
$$

and the Schrödinger equation for the leptonic wave function (with the leptonic hamiltonian $\hat{H}_{l}$ ) is solved for

*This article is dedicated to the memory of Professor Jacek Rychlewski various, fixed positions of the nuclei, giving the leptonic (or electronic) energy, $E_{l}$ dependent on $\mathbf{R}$

$$
\hat{H}_{l} \Psi_{l}(\mathbf{R}, \mathbf{r})=E_{l}(\mathbf{R}) \Psi_{l}(\mathbf{R}, \mathbf{r}),
$$

and providing the potential energy for nuclear function, which can be obtained by solving of the following equation:

$$
\left[\hat{T}_{n}(\mathbf{R})+E_{l}(\mathbf{R})+E_{a d}(\mathbf{R})\right] \Psi_{n}(\mathbf{R})=E \Psi_{n}(\mathbf{R}) .
$$

The adiabatic correction is expectation value of the $\hat{T}_{n}$ operator

$$
E_{a d}(\mathbf{R})=\left\langle\Psi_{l}(\mathbf{R}, \mathbf{r})\left|\hat{T}_{n}(\mathbf{R})\right| \Psi_{l}(\mathbf{R}, \mathbf{r})\right\rangle
$$

and measures the changes of leptonic wave function, induced by moving the nuclei from their current positions. It is not trivial to calculate $E_{a d}$ for all but atomic systems and it is usually neglected within the Born-Oppenheimer approximation. This is justified, as the values of adiabatic correction are usually much smaller than these of leptonic energies. In most calculations, carried out in practice, they are even within the error margins of $E_{l}$, as the accuracy of solutions of equation (3) is too low. However, the recent progress in development of computational methods, achieved in the group of professor Rychlewski, made it possible to obtain electronic energies having spectroscopic accuracy (i.e. with the error not exceeding a few microhartrees) for 
3- and 4-electron molecules (see [1] for a review). If the results of theoretical studies are to be compared with precise experimental measurements, the adiabatic correction can no longer be omitted.

The traditional way of calculation of $E_{a d}$ requires explicit separation of center of mass coordinates, which becomes very complicated beyond 2 nuclei [2]. Internal coordinates, mixing electronic and nuclear degrees of freedom, are introduced by that procedure. On the other hand, a pragmatic method has been proposed, in which the adiabatic correction is computed directly in the laboratory coordinate frame $[3,4]$. The equivalence of both approaches has been proven by Kutzelnigg [5]. The latter approach of calculation of $E_{a d}$ has been named as the BornHandy method. It provides a general framework, which can be applied for molecules of any geometry and was already used in practice with higly accurate explictly correlated Gaussian (ECG) wave functions [6]. It will be briefly discussed in the next section. Atomic units are used in the whole text.

\section{METHOD}

As no internal coordinates are introduced in the Born-Handy method, the adiabatic correction can be written as the sum of atomic contributions:

$$
E_{a d}=\sum_{A=1}^{N_{\text {nuc }}}-\frac{1}{2 M_{A}} \Delta_{A},
$$

where $\Delta_{A}$ are the expectation values of $\nabla^{2}$ operators, related to the coordinates of respective nuclei. It should be pointed out, that such a (formal) partitioning is related only to the concrete procedure, which is used in the present work and has nothing to do with a physical interpretation of the components of $E_{a d}$. In the cartesian coordinate frame, which is most convenient for calculations with ECG functions, each $\Delta_{A}$ integral is the following sum:

$$
\begin{gathered}
\Delta_{A}=\Delta_{x, A}+\Delta_{y, A}+\Delta_{z, A}= \\
=\left\langle\Psi_{l}\left|\frac{\partial^{2}}{\partial x_{A}^{2}}\right| \Psi_{l}\right\rangle+\left\langle\Psi_{l}\left|\frac{\partial^{2}}{\partial y_{A}^{2}}\right| \Psi_{l}\right\rangle+\left\langle\Psi_{l}\left|\frac{\partial^{2}}{\partial z_{A}^{2}}\right| \Psi_{l}\right\rangle .
\end{gathered}
$$

The integrals $\Delta_{x, A}, \Delta_{y, A}$ and $\Delta_{z, A}$ are dependent on the rotation of coordinate frame, while $\Delta_{A}$ is invariant.

Two similar algorithms, based on the numerical approximations of derivatives of the wave function (discussed in many textbooks of numerical methods),

$$
\frac{\partial \Psi_{l}(Q)}{\partial Q} \approx \frac{\Psi_{l}(Q+h)+\Psi_{l}(Q-h)}{2 h}
$$

and

$$
\frac{\partial^{2} \Psi_{l}(Q)}{\partial Q^{2}} \approx \frac{\Psi_{l}(Q+h)+\Psi_{l}(Q-h)-2 \Psi_{l}(Q)}{h^{2}},
$$

have been developed and applied in the calculations of $E_{a d}$ with ECG functions. The symbol $Q$ in Eqs. (8) and (9) denotes the $x, y$ or $z$ coordinate of a chosen nucleus. The respective expectation value of operator of 2'nd derivative will be denoted as

$$
\Delta_{Q}=\left\langle\Psi_{l} \mid \frac{\partial^{2} \Psi_{l}(Q)}{\partial Q^{2}}\right\rangle .
$$

The differentiation of normalization condition of the electronic wave function over $Q$ leads to another well known relation

$$
\left\langle\Psi_{l} \mid \frac{\partial^{2} \Psi_{l}(Q)}{\partial Q^{2}}\right\rangle=-\left\langle\frac{\partial \Psi_{l}(Q)}{\partial Q} \mid \frac{\partial \Psi_{l}(Q)}{\partial Q}\right\rangle .
$$

This relation, combined with equation (8) [7], leads to the approximation proposed by Cencek and Kutzelnigg. The integration over coordinates of light particles gives

$$
\Delta_{Q}^{(1)}=\frac{S_{ \pm}-1}{2 h^{2}} .
$$

This formula is also implemented in the PSI3 quantum chemistry code [8] for the Hartree-Fock and CI wave functions. Multiplication of Eq. (9) by $\Psi_{l}^{*}$ and similar integration gives the formula used recently by the present author $[9,10]$,

$$
\Delta_{Q}^{(2)}=\frac{S_{+}+S_{-}-2}{h^{2}} .
$$

Symbols $S_{+}, S_{-}$and $S_{ \pm}$denote the overlap integrals between leptonic wave functions of the system with a little different geometries, i.e. the chosen coordinate $(Q)$ of a nucleus is shifted by $h$ :

$$
\begin{aligned}
& S_{+}=\left\langle\Psi_{l}(Q) \mid \Psi_{l}(Q+h)\right\rangle, \\
& S_{-}=\left\langle\Psi_{l}(Q) \mid \Psi_{l}(Q-h)\right\rangle,
\end{aligned}
$$

and

$$
S_{ \pm}=\left\langle\Psi_{l}(Q-h) \mid \Psi_{l}(Q+h)\right\rangle .
$$

The leading term of error in approximations (12) and (13) is proportional to $h^{2}$. Only one overlap integral is needed for formula (12), while (13) requires two integrals, but their calculation is cheaper than solving the leptonic Schrödinger equation for $Q+h$ and $Q-h$, which is needed in both methods. The goal of present work is construction of an algorithm, which would use the same wave functions (for $Q, Q+h$ and $Q-h)$, but with a faster decreasing error. 
Let us consider the $\Delta Q$ integral for an isolated atom. Shift of the nucleus results in a translation of the whole wave function, so

$$
S_{+}(h)=S_{-}(h)=S_{ \pm}\left(\frac{h}{2}\right) .
$$

Equation (12) becomes equivalent with (13) for halved shift $h$, giving

$$
\Delta_{Q}^{(1)}\left(\frac{h}{2}\right)=\Delta_{Q}^{(2)}(h) .
$$

Therefore, the leading component of error of the approximation $\Delta_{Q}^{(1)}$ is 4 times larger than that of $\Delta_{Q}^{(2)}$, for a given $h$. It can be eliminated in the following expression

$$
\Delta_{Q}^{(3)}=\frac{4 \Delta_{Q}^{(2)}-\Delta_{Q}^{(1)}}{3}=\frac{8\left(S_{+}+S_{-}\right)-S_{ \pm}-15}{6 h^{2}} .
$$

For atoms, the error of approximation (19) decreases as $h^{4}$. However, moving a single nucleus in a molecule causes more complicated changes of the structure of wave function of light particles. Application of formula (19) requires then a silent assumption, that equation (17) is approximately fulfilled and the term of error, proportional to $h^{2}$, is largely cancelled.

\section{TEST CALCULATIONS}

In order to check the convergence patterns and final accuracy of $\Delta_{A}$ integrals, test calculations have been carried out for normal and positronic atoms and molecules containing up to 5 light particles. All systems were in their ground states. The results given by well grounded Eqs. (12) and (13) are compared with these of formula (19).

The leptonic wave functions of all systems, besides of the hydrogen atom, were linear combinations of explicitly correlated Gaussian (ECG) functions

$$
\Psi_{l}=\sum_{l} C_{l} \hat{A} \hat{P} e^{-\sum_{i=1}^{N} \alpha_{i}^{(I)}\left(\mathbf{r}_{i}-\mathbf{R}_{i}^{(I)}\right)^{2}-\sum_{i>j}^{N} \beta_{i j}^{(I)}\left(\mathbf{r}_{i}-\mathbf{r}_{j}\right)^{2}} \Theta_{S, M_{S}},
$$

with optimized nonlinear parameters $\alpha_{i}^{(I)}, \beta_{i}^{(I)}$ and $\mathbf{R}_{i}^{(I)}$. $\Theta_{S, M_{S}}$ is the spin function, $\hat{A}$ is the antisymmetrizer, which interchanges indistinguishable particles, while $\hat{P}$ ensures proper spatial symmetry of the wave function.

All calculations have been carried out in double precision. The mantissa of a double precision variable contains 53 bits. The numerical differentiation leads to a loss of accuracy, as numbers having similar values are substracted. In this operation, the leading significant bits are lost. Another source of uncertainty is the numerical noise, resulting from accumulating round-off errors and giving pseudorandom least significant bits in the variable. They are dependent on the processor type, compiler options etc. In order to estimate the number of certain significant bits ( $N$ bits), the overlap integral of leptonic wave function with itself

$$
S_{0}=\left\langle\Psi_{l}(Q) \mid \Psi_{l}(Q)\right\rangle
$$

was computed. Without the noise, it would be equal to one. In reality, its value differs slightly from one, and

$$
N_{\text {bits }}=\left[-\log _{2}\left|1-S_{0}\right|\right]
$$

where square brackets denote the integer part of a number.

For each system, the convergence $\Delta_{Q}$ of was studied, by performing calculations with different shifts $h$, starting from 0.032 and halving it, until $h<0.00002$. The numbers of stable and lost bits were calculated in each step. The leading bits of results were considered stable, if they did not change in two subsequent steps,

$$
\text { stable }=\left[\log _{2}\left|\frac{\Delta_{Q}(h)}{\Delta_{Q}(h)-\Delta_{Q}(2 h)}\right|\right]
$$

while the number of lost bits resulted from the substraction of similar numbers and was calculated as

$$
\operatorname{loss}(1)=\log _{2} \frac{1}{1-S_{ \pm}}
$$

for Eq. (12),

$$
\operatorname{los}(2)=\log _{2} \frac{2}{2-S_{+}-S_{-}}
$$

for Eq. (13) and

$$
\operatorname{loss}(3)=\log _{2} \frac{16}{15+S_{ \pm}-8\left(S_{+}+S_{-}\right)}
$$

for Eq. (19). The sum of numbers of stable and lost bits could not exceed the number of noise-free bits. If it did, then the extra accuracy was assumed to be a coincidence and the number of stable bits was assumed to be the difference between $N_{\text {bits }}$ and the number of lost bits. For convenience, the numbers of bits were also transformed to the numbers of decimal digits, using the formula decimal $=\log _{10} 2^{\text {bits }}$ (this is the reason of some inaccuracies, as the digits were not counted explicitly, but their number was calculated from the respective number of bits). Both numbers are given in the columns of tables, labeled "stable" and "loss", in the form bits/decimal digits.

\section{III.1. Atoms}

The calculations of integrals for atoms were an important part of the present study, as they offer a possibility to check independently the results, using analytical values. $\Delta_{x, A}$, $\Delta_{y, A}$ and $\Delta_{z, A}$ are equal, so that only one integral was com- 
Table 1. H, analytical wave function; $E_{l}=-0.5, \Delta_{H}=-1, S_{0}=1, N_{\text {bits }}=53$

\begin{tabular}{l|cccc|cccc}
\hline \multicolumn{1}{c|}{$h$} & $-\Delta_{\mathrm{H}}^{(2)}$ & stable & error & loss & $-\Delta_{\mathrm{H}}^{(3)}$ & stable & error & loss \\
\hline 0.032 & 0.9997483257 & & $2.517 \mathrm{e}-04$ & $13 / 3$ & 0.9999943464 & & $5.654 \mathrm{e}-06$ & $13 / 3$ \\
0.016 & 0.9999365434 & $12 / 3$ & $6.346 \mathrm{e}-05$ & $15 / 4$ & 0.9999992827 & $17 / 5$ & $7.174 \mathrm{e}-07$ & $15 / 4$ \\
0.008 & 0.9999840681 & $14 / 4$ & $1.593 \mathrm{e}-05$ & $17 / 5$ & 0.9999999096 & $20 / 6$ & $9.036 \mathrm{e}-08$ & $17 / 5$ \\
0.004 & 0.9999960085 & $16 / 4$ & $3.991 \mathrm{e}-06$ & $19 / 5$ & 0.9999999886 & $23 / 6$ & $1.135 \mathrm{e}-08$ & $19 / 5$ \\
0.002 & 0.9999990011 & $18 / 5$ & $9.989 \mathrm{e}-07$ & $21 / 6$ & 0.9999999986 & $26 / 7$ & $1.410 \mathrm{e}-09$ & $21 / 6$ \\
0.001 & 0.9999997503 & $20 / 6$ & $2.497 \mathrm{e}-07$ & $23 / 6$ & 1.0000000001 & $29 / 8$ & $1.400 \mathrm{e}-10$ & $23 / 6$ \\
0.0005 & 0.9999999389 & $22 / 6$ & $6.114 \mathrm{e}-08$ & $25 / 7$ & 1.0000000010 & $28 / 8$ & $1.030 \mathrm{e}-09$ & $25 / 7$ \\
0.00025 & 0.9999999762 & $24 / 7$ & $2.384 \mathrm{e}-08$ & $27 / 8$ & 0.9999999833 & $25 / 7$ & $1.674 \mathrm{e}-08$ & $27 / 8$ \\
0.000125 & 0.9999999975 & $24 / 7$ & $2.520 \mathrm{e}-09$ & $29 / 8$ & 0.9999999975 & $24 / 7$ & $2.520 \mathrm{e}-09$ & $29 / 8$ \\
$6.25 \mathrm{e}-05$ & 0.9999999975 & $22 / 6$ & $2.520 \mathrm{e}-09$ & $31 / 9$ & 0.9999998838 & $22 / 6$ & $1.162 \mathrm{e}-07$ & $31 / 9$ \\
$3.125 \mathrm{e}-05$ & 0.9999996564 & $20 / 6$ & $3.436 \mathrm{e}-07$ & $33 / 9$ & 0.9999994290 & $20 / 6$ & $5.710 \mathrm{e}-07$ & $33 / 9$ \\
\hline
\end{tabular}

Table 2. He, 100 ECG functions; $E_{l}=-2.903724347, \Delta_{\mathrm{He}}=-6.12558815759, S_{0}=1.000000000000013, N_{\text {bits }}=46$

\begin{tabular}{l|cccc|cccc}
\hline \multicolumn{1}{c|}{$h$} & $-\Delta_{\mathrm{He}}^{(2)}$ & stable & error & loss & $-\Delta_{\mathrm{He}}^{(3)}$ & stable & error & loss \\
\hline 0.032 & 6.1186495985 & & $6.939 \mathrm{e}-03$ & $10 / 3$ & 6.1253305342 & & $2.576 \mathrm{e}-04$ & $10 / 3$ \\
0.016 & 6.1238290151 & $10 / 3$ & $1.759 \mathrm{e}-03$ & $12 / 3$ & 6.1255554873 & $14 / 4$ & $3.267 \mathrm{e}-05$ & $12 / 3$ \\
0.008 & 6.1251452889 & $12 / 3$ & $4.429 \mathrm{e}-04$ & $14 / 4$ & 6.1255840468 & $17 / 5$ & $4.111 \mathrm{e}-06$ & $14 / 4$ \\
0.004 & 6.1254770485 & $14 / 4$ & $1.111 \mathrm{e}-04$ & $16 / 4$ & 6.1255876351 & $20 / 6$ & $5.225 \mathrm{e}-07$ & $16 / 4$ \\
0.002 & 6.1255603261 & $16 / 4$ & $2.783 \mathrm{e}-05$ & $18 / 5$ & 6.1255880854 & $23 / 6$ & $7.215 \mathrm{e}-08$ & $18 / 5$ \\
0.001 & 6.1255811057 & $18 / 5$ & $7.052 \mathrm{e}-06$ & $20 / 6$ & 6.1255880324 & $26 / 7$ & $1.252 \mathrm{e}-07$ & $20 / 6$ \\
0.0005 & 6.1255863075 & $20 / 6$ & $1.850 \mathrm{e}-06$ & $22 / 6$ & 6.1255880404 & $24 / 7$ & $1.172 \mathrm{e}-07$ & $22 / 6$ \\
0.00025 & 6.1255861610 & $22 / 6$ & $1.997 \tilde{\mathrm{e}}-06$ & $24 / 7$ & 6.1255861112 & $21 / 6$ & $2.046 \mathrm{e}-06$ & $24 / 7$ \\
0.000125 & 6.1255924067 & $19 / 5$ & $4.249 \mathrm{e}-06$ & $26 / 7$ & 6.1255944956 & $19 / 5$ & $6.338 \mathrm{e}-06$ & $26 / 7$ \\
$6.25 \mathrm{e}-05$ & 6.1255938135 & $18 / 5$ & $5.656 \mathrm{e}-06$ & $28 / 8$ & 6.1255943820 & $18 / 5$ & $6.224 \mathrm{e}-06$ & $28 / 8$ \\
$3.125 \mathrm{e}-05$ & 6.1256037043 & $16 / 4$ & $1.555 \mathrm{e}-05$ & $30 / 9$ & 6.1256068875 & $16 / 4$ & $1.873 \mathrm{e}-05$ & $30 / 9$ \\
\hline
\end{tabular}

Table 3. PsH, 768 ECG functions; $E_{l}=-0.78919627, \Delta_{H}=-1.19821626758, S_{0}=0.9999999999999305, N_{\text {bits }}=43$

\begin{tabular}{l|cccc|cccc}
\hline \multicolumn{1}{c|}{$h$} & $-\Delta_{\mathrm{H}}^{(2)}$ & stable & error & loss & $-\Delta_{\mathrm{H}}^{(3)}$ & stable & error & loss \\
\hline 0.032 & 1.1979003444 & & $3.159 \mathrm{e}-04$ & $12 / 3$ & 1.1982098962 & & $6.371 \mathrm{e}-06$ & $13 / 3$ \\
0.016 & 1.1981366847 & $12 / 3$ & $7.958 \mathrm{e}-05$ & $14 / 4$ & 1.1982154649 & $17 / 5$ & $8.027 \mathrm{e}-07$ & $15 / 4$ \\
0.008 & 1.1981963053 & $14 / 4$ & $1.996 \mathrm{e}-05$ & $16 / 4$ & 1.1982161788 & $20 / 6$ & $8.882 \mathrm{e}-08$ & $17 / 5$ \\
0.004 & 1.1982112657 & $16 / 4$ & $5.002 \mathrm{e}-06$ & $18 / 5$ & 1.1982162525 & $23 / 6$ & $1.503 \mathrm{e}-08$ & $19 / 5$ \\
0.002 & 1.1982149994 & $18 / 5$ & $1.268 \mathrm{e}-06$ & $20 / 6$ & 1.1982162440 & $22 / 6$ & $2.358 \mathrm{e}-08$ & $21 / 6$ \\
0.001 & 1.1982159045 & $20 / 6$ & $3.631 \mathrm{e}-07$ & $22 / 6$ & 1.1982162063 & $20 / 6$ & $6.132 \mathrm{e}-08$ & $23 / 6$ \\
0.0005 & 1.1982159665 & $19 / 5$ & $3.011 \mathrm{e}-07$ & $24 / 7$ & 1.1982159869 & $18 / 5$ & $2.807 \mathrm{e}-07$ & $25 / 7$ \\
0.00025 & 1.1982224493 & $17 / 5$ & $6.182 \mathrm{e}-06$ & $26 / 7$ & 1.1982246093 & $16 / 4$ & $8.342 \mathrm{e}-06$ & $27 / 8$ \\
0.000125 & 1.1982624812 & $14 / 4$ & $4.621 \mathrm{e}-05$ & $28 / 8$ & 1.1982758110 & $14 / 4$ & $5.954 \mathrm{e}-05$ & $29 / 8$ \\
$6.25 \mathrm{e}-05$ & 1.1983526065 & $13 / 3$ & $1.363 \mathrm{e}-04$ & $30 / 9$ & 1.1983827335 & $12 / 3$ & $1.665 \mathrm{e}-04$ & $31 / 9$ \\
$3.125 \mathrm{e}-05$ & 1.1989886843 & $10 / 3$ & $7.724 \mathrm{e}-04$ & $32 / 9$ & 1.1992005966 & $10 / 3$ & $9.843 \mathrm{e}-04$ & $33 / 9$ \\
\hline
\end{tabular}


puted and multipled by 3 . Only the results obtained with Eq. (13) are given in Table 1-4, due to equality (18).

For the ground state of hydrogen atom, the analytical wave function has been used. The overlap integral was also calculated analytically [11] $\left(S(h)=\left\langle\Psi_{l}(Q) \mid \Psi_{l}(Q+h)\right\rangle=\right.$ $\left.=\left(1+h+h^{3} / 3\right) e^{-h}\right)$, so that it can be safely assumed, that no numerical noise was present. As can be seen in Table 1, faster convergence of the value of $\Delta_{A}$ is achieved with equation (19). The most accurate result has been obtained for $h=0.001$, where also the largest number of stable bits has been reached. The error is even smaller than suggested by the number of stable bits. Formula (13) gives the smallest error for $h=0.000125$, but maximal number of stable bits is reached for $h=0.00025$. This discrepancy may be a coincidence, but may also appear, because the calculations were carried out on the Intel i486 processor, whose floating point unit registers have the internal precision of 80 bits (with 64 bits of mantissa). Even if the "illegally" more accurate value would be accepted, its error is anyway one order of magnitude larger than that given by Eq. (19).

In all other cases, the wave functions are approximations given by equation (20). The numerical noise causes, that the best accuracy of $E_{a d}$ is lower than for $H$ atom and is reached at larger $h$. The calculations have been carried out for atoms of helium, positronium hydride and positronic lithium. Expansion lengths, leptonic energies, reference values of $\Delta_{A}$ integrals (calculated analytically), $S_{0}$ integrals and numbers of certain significant bits are given in the captions of respective tables. The accuracy of wave functions decreases with the system size, but they can be all considered to be of high quality. The errors of leptonic energies amount to about 10 nanohartrees for $\mathrm{He}$, 0.7 microhartree for PsH and no more than 5 microhartrees for $\mathrm{PsLi}^{+}$[12]. The energy of the latter system is the lowest published to date, being about $10 \mu$ hartrees below the recent result of Mitroy [13].

The same convergence pattern is repeated for all systems - the values of $\Delta_{A}$ increase to the most accurate one, which is reached at $h=0.0005$ with formula (13) and at $h=0.002$ (0.004 for PsH) with formula (19). Further decreasing of displacements causes the loss of accuracy, due to the numerical instability. Formula (19) gives 2-5 more stable bits ( 1 decimal digit) and error smaller by order of magnitude than (12) and (13). The numbers of bits free of the numerical noise are smaller than 53 and decrease with the number of light particles and expansion length.

\section{III.2. Diatomic systems}

The calculations of $\Delta_{A}$ integrals for molecules provided the real test of new algorithm, which is not necessarily correct, as Eq. (17) is not automatically fulfilled. They have been carried out for the hydrogen molecule (with two wave functions of different accuracy), helium dimer and positronic lithium hydride molecule, at equilibrium internuclear distances of 1.4011 bohr for $\mathrm{H}_{2}, 5.6$ bohr for $\mathrm{He} \cdots \mathrm{He}$ and $3.365 \mathrm{bohr}$ for $\mathrm{e}^{+} \mathrm{LiH}$. The wave function of positronic $\mathrm{LiH}$ was the least accurate of all systems - the energy error is estimated to be of the order of 0.1 millihartree [12].

No analytical reference results could be obtained for molecular systems. The accuracy of $\Delta_{Q}$ was controlled in two ways. First, the stable bits were counted, similarly as for atoms. The results were also compared with the values provided by extrapolation procedure, which is equivalent with Eq. (19) for atoms:

$$
\Delta_{Q}^{(4)}=\frac{4 \Delta_{Q}^{(1)}-\Delta_{Q}^{(1)}(2 h)}{3},
$$

Table 5. $\mathrm{H}_{2}, 75$ ECG functions; $E_{l}=-1.1744751, S_{0}=0.9999999999999685, N_{\text {bits }}=44$

\begin{tabular}{l|cll|lll|lll|l}
\hline \multicolumn{1}{c|}{$h$} & $-\Delta_{\mathrm{H}}^{(1)}$ & stable & loss & $-\Delta_{\mathrm{H}}^{(2)}$ & stable & loss & $-\Delta_{\mathrm{H}}^{(3)}$ & stable & loss & $-\Delta_{\mathrm{H}}^{(4)}$ \\
\hline 0.032 & 0.9565258 & & $11 / 3$ & 0.9574761 & & $13 / 4$ & 0.9577929 & & $13 / 4$ & \\
0.016 & 0.9575013 & $9 / 2$ & $13 / 4$ & 0.9577442 & $11 / 3$ & $15 / 5$ & 0.9578252 & $14 / 4$ & $15 / 5$ & 0.9578265 \\
0.008 & 0.9577506 & $11 / 3$ & $15 / 5$ & 0.9578120 & $13 / 3$ & $17 / 5$ & 0.9578324 & $17 / 5$ & $17 / 5$ & 0.9578336 \\
0.004 & 0.9578135 & $13 / 3$ & $17 / 5$ & 0.9578290 & $15 / 4$ & $19 / 6$ & 0.9578341 & $19 / 5$ & $19 / 6$ & 0.9578345 \\
0.002 & 0.9578296 & $15 / 4$ & $19 / 6$ & 0.9578335 & $17 / 5$ & $21 / 6$ & 0.9578347 & $20 / 6$ & $21 / 6$ & 0.9578349 \\
0.001 & 0.9578330 & $18 / 5$ & $21 / 6$ & 0.9578339 & $21 / 6$ & $23 / 7$ & 0.9578342 & $20 / 6$ & $23 / 7$ & 0.9578341 \\
0.0005 & 0.9578341 & $19 / 5$ & $23 / 7$ & 0.9578357 & $18 / 5$ & $25 / 8$ & 0.9578363 & $18 / 5$ & $25 / 8$ & 0.9578345 \\
0.00025 & 0.9578322 & $18 / 5$ & $25 / 8$ & 0.9578371 & $18 / 5$ & $27 / 8$ & 0.9578388 & $18 / 5$ & $27 / 8$ & 0.9578316 \\
0.000125 & 0.9578405 & $16 / 4$ & $27 / 8$ & 0.9578352 & $16 / 4$ & $29 / 9$ & 0.9578334 & $16 / 4$ & $29 / 9$ & 0.9578432 \\
$6.25 \mathrm{e}-05$ & 0.9578258 & $15 / 4$ & $29 / 9$ & 0.9577965 & $14 / 4$ & $31 / 9$ & 0.9577868 & $14 / 4$ & $31 / 9$ & 0.9578209 \\
$3.125 \mathrm{e}-05$ & 0.9578342 & $14 / 4$ & $31 / 9$ & 0.9579019 & $12 / 3$ & $33 / 10$ & 0.9579244 & $12 / 3$ & $33 / 10$ & 0.9578370 \\
\hline
\end{tabular}


Table 6. $\mathrm{H}_{2}$ molecule, 837 ECG functions; $E_{l}=-1.1744759312, S_{0}=0.9999999999998710, N_{\text {bits }}=42$

\begin{tabular}{l|ccl|ccc|ccc|c}
\hline \multicolumn{1}{c|}{$h$} & $-\Delta_{\mathrm{H}}^{(1)}$ & stable & loss & $-\Delta_{\mathrm{H}}^{(2)}$ & stable & loss & $-\Delta_{\mathrm{H}}^{(3)}$ & stable & loss & $-\Delta_{\mathrm{H}}^{(4)}$ \\
\hline 0.032 & 0.9570840 & & $11 / 3$ & 0.9580366 & & $13 / 4$ & 0.9583542 & & $13 / 4$ & \\
0.016 & 0.9580618 & $9 / 2$ & $13 / 4$ & 0.9583053 & $11 / 3$ & $15 / 5$ & 0.9583865 & $14 / 4$ & $15 / 5$ & 0.9583877 \\
0.008 & 0.9583116 & $11 / 3$ & $15 / 5$ & 0.9583731 & $13 / 3$ & $17 / 5$ & 0.9583936 & $17 / 5$ & $17 / 5$ & 0.9583948 \\
0.004 & 0.9583747 & $13 / 3$ & $17 / 5$ & 0.9583901 & $15 / 4$ & $19 / 6$ & 0.9583953 & $19 / 5$ & $19 / 6$ & 0.9583957 \\
0.002 & 0.9583905 & $15 / 4$ & $19 / 6$ & 0.9583943 & $17 / 5$ & $21 / 6$ & 0.9583955 & $21 / 6$ & $21 / 6$ & 0.9583958 \\
0.001 & 0.9583943 & $17 / 5$ & $21 / 6$ & 0.9583957 & $19 / 5$ & $23 / 7$ & 0.9583962 & $20 / 6$ & $23 / 7$ & 0.9583956 \\
0.0005 & 0.9583966 & $18 / 5$ & $23 / 7$ & 0.9583982 & $18 / 5$ & $25 / 8$ & 0.9583987 & $18 / 5$ & $25 / 8$ & 0.9583973 \\
0.00025 & 0.9583976 & $18 / 5$ & $25 / 8$ & 0.9584106 & $16 / 4$ & $27 / 8$ & 0.9584149 & $15 / 4$ & $27 / 8$ & 0.9583980 \\
0.000125 & 0.9584047 & $16 / 4$ & $27 / 8$ & 0.9584728 & $13 / 3$ & $29 / 9$ & 0.9584955 & $13 / 3$ & $29 / 9$ & 0.9584070 \\
$6.25 \mathrm{e}-05$ & 0.9584114 & $14 / 4$ & $29 / 9$ & 0.9585891 & $12 / 3$ & $31 / 9$ & 0.9586483 & $12 / 3$ & $31 / 9$ & 0.9584136 \\
$3.125 \mathrm{e}-05$ & 0.9588280 & $11 / 3$ & $31 / 9$ & 0.9588480 & $10 / 3$ & $33 / 10$ & 0.9588548 & $10 / 3$ & $33 / 10$ & 0.9589669 \\
\hline
\end{tabular}

Table 7. Helium dimer, 2382 ECG functions; $E_{l}=-5.80748355, S_{0}=1.000000000002466, N_{\text {bits }}=38$

\begin{tabular}{l|cll|lll|lll|l}
\hline \multicolumn{1}{c|}{$h$} & $-\Delta_{\mathrm{He}}^{(1)}$ & stable & loss & $-\Delta_{\mathrm{He}}^{(2)}$ & stable & loss & $-\Delta_{\mathrm{He}}^{(3)}$ & stable & loss & $-\Delta_{\mathrm{He}}^{(4)}$ \\
\hline 0.032 & 6.0983722 & & $8 / 2$ & 6.1184141 & & $10 / 3$ & 6.1250948 & & $10 / 3$ & \\
0.016 & 6.1184141 & $8 / 2$ & $10 / 3$ & 6.1235933 & $10 / 3$ & $12 / 4$ & 6.1253197 & $14 / 4$ & $12 / 4$ & 6.1250947 \\
0.008 & 6.1235933 & $10 / 3$ & $12 / 4$ & 6.1249094 & $12 / 3$ & $14 / 4$ & 6.1253481 & $17 / 5$ & $14 / 4$ & 6.1253197 \\
0.004 & 6.1249095 & $12 / 3$ & $14 / 4$ & 6.1252410 & $14 / 4$ & $16 / 5$ & 6.1253515 & $20 / 6$ & $16 / 5$ & 6.1253482 \\
0.002 & 6.1252408 & $14 / 4$ & $16 / 5$ & 6.1253232 & $16 / 4$ & $18 / 5$ & 6.1253507 & $21 / 6$ & $18 / 5$ & 6.1253512 \\
0.001 & 6.1253236 & $16 / 4$ & $18 / 5$ & 6.1253442 & $18 / 5$ & $20 / 6$ & 6.1253510 & $19 / 5$ & $20 / 6$ & 6.1253512 \\
0.0005 & 6.1253447 & $18 / 5$ & $20 / 6$ & 6.1253308 & $17 / 5$ & $22 / 7$ & 6.1253261 & $17 / 5$ & $22 / 7$ & 6.1253517 \\
0.00025 & 6.1253420 & $17 / 5$ & $22 / 7$ & 6.1253960 & $15 / 4$ & $24 / 7$ & 6.1254140 & $15 / 4$ & $24 / 7$ & 6.1253411 \\
0.000125 & 6.1252862 & $15 / 4$ & $24 / 7$ & 6.1253025 & $13 / 3$ & $26 / 8$ & 6.1253079 & $13 / 3$ & $26 / 8$ & 6.1252677 \\
$6.25 \mathrm{e}-05$ & 6.1248507 & $13 / 3$ & $26 / 8$ & 6.1231441 & $11 / 3$ & $28 / 8$ & 6.1225753 & $11 / 3$ & $28 / 8$ & 6.1247055 \\
$3.125 \mathrm{e}-05$ & 6.1245067 & $11 / 3$ & $28 / 8$ & 6.1191938 & $9 / 2$ & $30 / 9$ & 6.1174233 & $9 / 2$ & $30 / 9$ & 6.1243920 \\
\hline
\end{tabular}

gives the error proportional to $h^{4}$ and is not based on a vague assumption, but requires additional evaluation of wave functions for $Q+2 h$ and $Q-2 h$.

If the molecule is placed along one of the coordinate frame axes, two components of $\Delta_{A}$ are to be computed parallel and perpendicular to the bond axis. In the present work, another orientation of the molecule has been chosen - the bond axis is a diagonal of the cube, whose 3 walls coincide with the coordinate frame planes. Only one $\Delta_{Q}$ integral is then needed, as $\Delta_{x, A}, \Delta_{y, A}$ and $\Delta_{z, A}$ are equal.

Due to the symmetry of hydrogen molecule and helium dimer, both atomic contributions are the same and only one is given in Tables 5-7. The symmetry projector in equation (20) doubles the numbers of "primitive" (i.e. non symmetry adapted) basis functions, but calculation of $\Delta_{Q}$ integrals was carried out with spatial symmetry disabled, so that the real expansion lengths amounted to 150 and 1674 for $\mathrm{H}_{2}$, and 4764 for He $\cdots$ He. The latter system was described with the reoptimized 2400-term function [14], from which a few basis functions, which did not lower the energy, have been removed.

The convergence patterns in molecular calculations agree with these observed for atoms, although the impro- vement is a little smaller. Formula (19) gives usually up to 2-3 more stable bits than (12) and (13), but there are some cases, in which those equations seem to work better. For the hydrogen molecule, described with the 75-term wave function, formula (13) seems to give 21 stable bits, while (19) provides only 20 bits. However, formula (27), which should be regarded as the most accurate one, gives the value of $\Delta_{H}$ closer to that of (19) and its six significant digits of the result are stable in a wide range of $h$. Another example of a "false stability" appears for the helium dimer with equation (12), where 6 significant digits do not change in the value of $\Delta_{\mathrm{He}}=6.12534$, when $h$ decreases from 0.0005 to 0.00025 . This calculation has been carried out on the edge of real precision of 38 bits ( 15 bits lost, due to the numerical noise!) and the 6 'th digit is incorrect - formulas (19) and (27) give a little larger $\Delta_{\mathrm{He}}=6.12535$. This example justifies the method of estimation of accuracy, which takes the number of certain bits in account and shows, that just counting the unchanging digits may be not sufficient.

The calculations for positronic lithium hydride molecule show, that a single value of $h$ for both atoms would be suboptimal. The LiH molecule is highly polar and the 
Table 8. Positronic LiH molecule, 2048 ECG functions; $E_{l}=-8.107864, S_{0}=.9999999999993515, N_{\text {bits }}=40$

\begin{tabular}{|c|c|c|c|c|c|c|c|c|c|c|}
\hline$h$ & $-\Delta_{\mathrm{Li}}^{(1)}$ & stable & loss & $-\Delta_{\mathrm{Li}}^{(2)}$ & stable & loss & $-\Delta_{\mathrm{Li}}^{(3)}$ & stable & loss & $-\Delta_{\mathrm{Li}}^{(4)}$ \\
\hline 0.032 & 15.1438384 & & $7 / 2$ & 15.2588572 & & $9 / 3$ & 15.2971968 & & $9 / 3$ & \\
\hline 0.016 & 15.2588505 & $7 / 2$ & $9 / 3$ & 15.2890412 & $8 / 2$ & $11 / 3$ & 15.2991048 & $12 / 3$ & $11 / 3$ & 15.2971879 \\
\hline 0.008 & 15.2890395 & $8 / 2$ & $11 / 3$ & 15.2967688 & $10 / 3$ & $13 / 4$ & 15.2993452 & $15 / 4$ & $13 / 4$ & 15.2991026 \\
\hline 0.004 & 15.2967684 & $10 / 3$ & $13 / 4$ & 15.2987234 & $12 / 3$ & $15 / 5$ & 15.2993750 & $18 / 5$ & $15 / 5$ & 15.2993447 \\
\hline 0.002 & 15.2987230 & $12 / 3$ & $15 / 5$ & 15.2992148 & $14 / 4$ & $17 / 5$ & 15.2993788 & $21 / 6$ & $17 / 5$ & 15.2993746 \\
\hline 0.001 & 15.2992151 & $14 / 4$ & $17 / 5$ & 15.2993340 & $16 / 4$ & $19 / 6$ & 15.2993736 & $21 / 6$ & $19 / 6$ & 15.2993791 \\
\hline 0.0005 & 15.2993365 & $16 / 4$ & $19 / 6$ & 15.2993497 & $19 / 5$ & $21 / 6$ & 15.2993541 & $19 / 5$ & $21 / 6$ & 15.2993770 \\
\hline 0.00025 & 15.2993784 & $18 / 5$ & $21 / 6$ & 15.2993641 & $17 / 5$ & $23 / 7$ & 15.2993594 & $17 / 5$ & $23 / 7$ & 15.2993924 \\
\hline 0.000125 & 15.2993011 & $17 / 5$ & $23 / 7$ & 15.2992115 & $15 / 4$ & $25 / 8$ & 15.2991815 & $15 / 4$ & $25 / 8$ & 15.2992754 \\
\hline $6.25 \mathrm{e}-05$ & 15.2993299 & $15 / 4$ & $25 / 8$ & 15.2992567 & $13 / 3$ & $27 / 8$ & 15.2992322 & $13 / 3$ & $27 / 8$ & 15.2993395 \\
\hline $3.125 \mathrm{e}-05$ & 15.2993633 & $13 / 3$ & $27 / 8$ & 15.2979549 & $11 / 3$ & $29 / 9$ & 15.2974853 & $11 / 3$ & $29 / 9$ & 15.2993744 \\
\hline$h$ & $-\Delta_{\mathrm{H}}^{(1)}$ & stable & loss & $-\Delta_{\mathrm{H}}^{(2)}$ & stable & loss & $-\Delta_{\mathrm{H}}^{(3)}$ & stable & loss & $-\Delta_{\mathrm{H}}^{(4)}$ \\
\hline 0.032 & 1.2879212 & & $10 / 3$ & 1.2889341 & & $12 / 4$ & 1.2892718 & & $13 / 4$ & \\
\hline 0.016 & 1.2889270 & $10 / 3$ & $12 / 4$ & 1.2891844 & $12 / 3$ & $14 / 4$ & 1.2892702 & $19 / 5$ & $15 / 5$ & 1.2892623 \\
\hline 0.008 & 1.2891826 & $12 / 3$ & $14 / 4$ & 1.2892474 & $14 / 4$ & $16 / 5$ & 1.2892690 & $20 / 6$ & $17 / 5$ & 1.2892678 \\
\hline 0.004 & 1.2892470 & $14 / 4$ & $16 / 5$ & 1.2892630 & $16 / 4$ & $18 / 5$ & 1.2892684 & $20 / 6$ & $19 / 6$ & 1.2892684 \\
\hline 0.002 & 1.2892634 & $16 / 4$ & $18 / 5$ & 1.2892665 & $18 / 5$ & $20 / 6$ & 1.2892675 & $19 / 5$ & $21 / 6$ & 1.2892689 \\
\hline 0.001 & 1.2892680 & $18 / 5$ & $20 / 6$ & 1.2892644 & $18 / 5$ & $22 / 7$ & 1.2892632 & $17 / 5$ & $23 / 7$ & 1.2892695 \\
\hline 0.0005 & 1.2892639 & $18 / 5$ & $22 / 7$ & 1.2892578 & $16 / 4$ & $24 / 7$ & 1.2892558 & $15 / 4$ & $25 / 8$ & 1.2892625 \\
\hline 0.00025 & 1.2892431 & $15 / 4$ & $24 / 7$ & 1.2892582 & $14 / 4$ & $26 / 8$ & 1.2892632 & $13 / 3$ & $27 / 8$ & 1.2892362 \\
\hline 0.000125 & 1.2893067 & $14 / 4$ & $26 / 8$ & 1.2894130 & $12 / 3$ & $28 / 8$ & 1.2894485 & $11 / 3$ & $29 / 9$ & 1.2893279 \\
\hline $6.25 \mathrm{e}-05$ & 1.2890619 & $12 / 3$ & $28 / 8$ & 1.2888515 & $10 / 3$ & $30 / 9$ & 1.2887813 & $9 / 2$ & $31 / 9$ & 1.2889803 \\
\hline $3.125 \mathrm{e}-05$ & 1.2892509 & $10 / 3$ & $30 / 9$ & 1.2859543 & $8 / 2$ & $32 / 10$ & 1.2848559 & $7 / 2$ & $33 / 10$ & 1.2893139 \\
\hline
\end{tabular}

presence of the positively charged positron, which is localized at the negative $(H)$ end [15], causes its additional polarization - the bond length increases and the electron density moves towards the $H$ nucleus. The lowest energy dissociation products of $e^{+} \mathrm{LiH}$ are positronium hydride and lithium cation. Close to the equilibrium distance, moving of the Li nucleus, surrounded by the compact distribution of its core electrons, gives most accurate approximation of $\Delta_{\mathrm{Li}}$ integral at $h=0.002$. On the other hand, $\Delta_{H}$ is very close to that of isolated $\mathrm{PsH}$ atom, which leptonic density is much more diffuse than that of $\mathrm{Li}^{+}$and the most accurate approximation is obtained at $h=0.004$.

\section{CONCLUSIONS}

The proposed method of calculation of adiabatic corrections, although not proven mathematically for molecules, seems to work well for all studied systems, which have different leptonic structures. It gives usually one additional stable significant digit, in comparison with Eqs. (12) and (13), using the same wave functions. This extra stability is not a spurious effect, as the results are close to these of formula (27), which error certainly decreases as $h^{-4}$. The most stable values of $\Delta_{A}$ are obtained for larger shifts. The recommended value of $h$ for the new algorithm is between 0.002 and 0.004 bohr.

One may ask, whether calculation of 6 instead of 5 significant decimal digits of the adiabatic correction is worth the effort of evaluation of additional overlap integrals for Eq. (19), or even wave functions for equation (27), if the result would be changed by a few nanohartrees. The answer is not unique. In most calculations, there would be no practical difference. Unfortunately, decreasing real precision is the price to be paid for the increasing accuracy of leptonic energies. The number of certain bits decreases with the expansion length of wave function (20) and with the number of light particles. Let us compare the two, 75and 837-term wave functions of hydrogen molecule, which give the electronic energies accurate to a fraction microhartree and nanohartree, respectively, but with 44 and 42 cer- 
tain bits. For the short function, the satisfactory accuracy of $E_{a d}$ would be about $0.1 \mu$ hartree. Assuming the proton mass equal to 1836.15267 a.u., the value of 0.0005217 hartree is obtained. It requires only 4 stable significant digits of $\Delta_{H}$, which can be easily calculated even with methods (12) or (13). However, for the long expansion we would like to compute the adiabatic correction with the accuracy comparable to that of electronic energy. The new method gives the value of 0.0005219585 hartree, with the last digit uncertain, which is acceptable, while two last digits would be uncertain, if one of older algorithms would be applied. In the case of larger systems, described with extensive wave functions, for which the loss of precision resulting from the numerical noise is significant, this additional significant digit may allow to avoid the time consuming calculation of $E_{a d}$ with extended precision variables. The obvious target could be the helium dimer, for which calculations with very long expansions (up to 4800 ECG functions) have been carried out recently [16].

Another area of practical application of the new algorithm are the studies of positronic systems, in which the positron is treated as a light pseudonucleus with the charge less than 1 [17], within a modified adiabatic approximation. Due to small positron mass, the contribution of $E_{a d}$ to the effective potential for positronic motion is comparable with that of electronic energy.

\section{Acknowledgement}

This study was supported by Wrocław University of Technology. Thanks are also due to Dr. Komasa from
Adam Mickiewicz University, for providing accurate electronic wave function of the helium dimer.

\section{References}

[1] J. Rychlewski, J. Komasa, in: Explicitly Correlated Wave Functions in Chemistry and Physics, Kluwer Academic Publishers 2003, pp. 91-137.

[2] R. D. Bardo, M. Wolfsberg, J. Chem. Phys. 68, 2686 (1978).

[3] H. Sellers, P. Pulay, Chem. Phys. Lett. 103, 463 (1984).

[4] N. C. Handy, Y. Yamaguchi, H. F. Schaefer III, J. Chem. Phys. 84, 4481 (1986).

[5] W. Kutzelnigg, Mol. Phys. 90, 909 (1997).

[6] J. Rychlewski, W. Cencek, in Explicitly Correlated Wave Functions in Chemistry and Physics, Kluwer Academic Publishers 2003, pp. 249-274.

[7] W. Cencek, W. Kutzelnigg, Chem. Phys. Lett. 266, 383 (1997).

[8] http://www.psicode.org and http://sourceforge.net/projects/psicode

[9] K. Strasburger, J. Phys. B 37, 4483 (2004).

[10] K. Strasburger, H. Chojnacki, A. Sokołowska, J. Phys. B38, 3091 (2005).

[11] H.-J. Glaeske, J. Reinhold, P. Volkmer, in Quantenchemie. Ein Lehrgang. Band 5. Ausgewählte mathematische Methoden der Chemie, VEB Deutscher Verlag der Wissenschaften, Berlin 1987, p. 567.

[12] K. Strasburger, CCP2 Workshop, Nottingham, 2-5.04.2006.

[13] J. Mitroy, Phys. Rev. A 70, 024502 (2004).

[14] J. Komasa, private communication.

[15] K. Strasburger, J. Chem. Phys. 111, 10555 (1999).

[16] W. Cencek, J. Komasa, K. Pachucki and K. Szalewicz, Phys. Rev. Lett 95, 233004 (2005).

[17] K. Strasburger, M. Wołcyrz, Mol. Phys., accepted for publication.

KRZYSZTOF STRASBURGER was born in 1966 in Wrocław and lives there up to date. He received his MSc in chemistry in 1990 and PhD in 1996 from Wrocław University of Technology. He works at Departament of Chemistry of Wrocław Univeristy of Technology, in the Molecular Modelling and Quantum Chemistry group. His scientific activity focuses on applications of explicitly correlated functions for studies of matter-antimatter interactions. 